\title{
Diagnosis DEl CONOCIMIENTO DE LA FAUNA DE OCTOCORALES (CNIDARIA, ANTHOZOA) DE LA REgIÓN NORDESTE DE BRASIL.
}

\author{
BRUNO T. SILVA \\ CARLOS D. PÉREZ ${ }^{1}$
}

G.P.A.-NEC-LACE. Depto. Zoologia-CCB-UFPE. Prof. Moraes Rego 1235, Cidade Universitária, CEP 50670-420, Recife-PE. Email: cdperez@ufpe.br

${ }^{1}$ Investigador del CNPq

\section{RESUMEN}

Los octocorales constituyen un grupo faunístico de notable presencia en las comunidades bentónicas por su belleza, diversidad, abundancia y las relaciones interespecíficas. La sistemática y la zoogeografía de los antozoos constituyen un interesante problema a analizar, ya que en el Atlántico Sur los registros son escasos y desactualizados, excepto para determinados órdenes como Actiniaria. La fauna de los octocorales del Atlántico Sudoccidental está pobremente conocida para la costa sudamericana desde Trinidad y Tobago hasta la foz del Río de la Plata en Argentina. Cuando se trabaja en la identificación y distribución de los organismos que ocurren en el litoral nordestino brasileiro, se observa una gran confusión. Por eso la necesidad de conocer la diversidad de los octocorales presentes en la costa de esa región, para evitar desencuentros de informaciones, y auxiliar en la identificación de las especies colectadas. Según el análisis bibliográfico realizado, fueron encontradas 59 especies descriptas para Brasil, de las cuales 25 se encuentran en la región Nordeste (Bahía 17, Pernambuco 8, Maranhão 6, Rio Grande do Norte 5, Paraíba 4, Piauí 1 y Sergipe sin registros), representando un $42,37 \%$. El porcentaje obtenido para las especies que ocurren en la región NE es un poco engañoso y merece una aclaración. De las 25 especies registradas, 17 están registradas para el estado de Bahía, principalmente en el Archipiélago de Abrolhos, lugar sumamente estudiado por investigadores del sur-sudeste brasilero; y estas investigaciones encubren el verdadero grado de conocimiento de la octofauna del $\mathrm{NE}$ que, excluyendo al estado de Bahía, representan el 25, 42 \%, colocando a dicha región como pobremente conocida. Actualmente están siendo desarrollados varios estudios con octocorales por el G.P.A. (Grupo de Pesquisa em Antozoários) de la UFPE, que dentro de sus líneas de investigación se encuentra la Sistemática, Taxonomía, Ecología y Filogenia de los octocorales del Nordeste brasilero.

Palabras clave: Anthozoa, Octocorallia, diversidad, Brasil, Nordeste

\section{RESUMO}

\section{Diagnose do conhecimento da fauna de octocorais (Cnidaria, Anthozoa) da região Nordeste do Brasil}

Os octocorais constituem um grupo faunístico de notável presença nas comunidades bentônicas por sua beleza, diversidade, abundância e as relações interespecíficas. A sistemática e a zoogeografia dos antozoários representam um interessante problema a analisar, já que no Atlântico Sul, os registros são escassos e desatualizados, exceto para determinadas ordens como Actiniaria. A 
fauna de octocorais no Atlântico Sul Ocidental é pobremente conhecida para a costa sul-americana desde Trinidad e Tobago, até o Rio da Prata, na Argentina. Ao se trabalhar na identificação e distribuição dos organismos que ocorrem no litoral nordestino, se observa uma grande confusão. Por isso a necessidade de conhecer a diversidade dos octocorais presentes na costa nordestina brasileira, para evitar desencontro de informações, e auxiliar na identificação das espécies coletadas. Em análise bibliográfica, foram encontradas 59 espécies descritas para o Brasil, dentre as quais, 25 encontram-se no Nordeste (Bahia 17, Pernambuco 8, Maranhão 6, Rio Grande do Norte 5, Paraíba 4, Piauí 1 e Sergipe sem registros), representando 42,37\%. A porcentagem obtida para as espécies que ocorrem na região NE é um pouco enganosa, e merece um esclarecimento. Das 25 espécies registradas, 17 ocorrem no estado da Bahia, principalmente no Arquipélago de Abrolhos, local sumamente estudado por pesquisadores do sul-sudeste brasileiro, e estas pesquisas encobrem o real grau de conhecimento da octofauna do NE que, tirando o estado da Bahia, representam 25,42\%, colocando a região como pobremente conhecida. Atualmente estão sendo desenvolvidos vários estudos com octocorais pelo G.P.A. (Grupo de Pesquisa em Antozoários) da UFPE, que entre suas linhas de pesquisa está a Sistemática, Taxonomia e Ecologia dos Octocorais do NE.

Palavras chave: Anthozoa, Octocorallia, Diversidade, Brasil, Nordeste

\begin{abstract}
Diagnosis of knowledge of the fauna of octocorals (Cnidaria, Anthozoa) of Northeastern region of Brazil.
\end{abstract}

The octocorals represent a faunistic group of great presence in benthic communities due to its beauty, diversity, abundance and interspecific relationships. The systematic and zoogeography of anthozoans are an interesting problem for analyzing because the records in the South Atlantic Ocean are scanty and not up to date, except for Order Actiniaria. The fauna of octocorals from South Atlantic Ocean is poorly known for the south American coast from Trinidad and Tobago to La Plata River.The taxonomy study and distribution of organisms from northeastern coast appear a real mess. For this reason it is necessary to know the diversity of octocorals present in the region avoiding loss of information and assisting in the identification of species. From bibliographic analyse, it was found 59 species occurring in Brazil, 25 of them are present in the Northeastern region (Bahia 17, Pernambuco 8, Maranhão 6, Rio Grande do Norte 5, Paraíba 4, Piauí 1 and Sergipe without records) representing 42,37\%. It is important to notice that from 25 species, 17 occur in state of Bahia, specially in Abrolhos Archipiel. This area is well studied by researches from the south and southeastern of Brazil. Excepting the state of Bahia, the percent of species known for the northeastern is $25,42 \%$ which makes this a poorly known area. Actually, there are in development several studies with octocorals for the G.P.A. (Research Group in Antozoans) from UFPE, and in its scope is the Sistematic, Taxonomy, Ecology and Filogeny of octocorals from Northeastern region.

Key words: Anthozoa, Octocorallia, Diversity, Brazil, Northeastern

\title{
INTRODUCCIÓN
}

Los octocorales constituyen un grupo faunístico de notable presencia en las comunidades bentónicas por su belleza, diversidad, abundancia y las relaciones interespecíficas (BRITO, 1993). Esta clase está representada por un gran número de formas que van desde incrustantes, filiformes 
y membranosas, hasta completas y elaboradas arquitecturas ramificadas. Su distribución está asociada a zonas de alta productividad, sirviendo de refugio y alimento a muchas especies que habitan su ambiente (PÉREZ, 1999).

La sistemática y la zoogeografía de los antozoos constituyem un interesante problema a analizar, ya que en el Océano Atlántico Sur, los registros son escasos y desactualizados (BAYER, 1959; CASTRO, 1990), excepto para determinados órdenes como los actiniarios.

Bayer (1981a) propone la clasificación de las áreas en cuatro niveles según el grado de conocimiento taxonómico:

1. Esencialmente conocido: áreas donde hay una rica literatura y la mayoría de las especies han sido adecuadamente descriptas.

2. Moderadamente bien conocido: áreas donde hay una amplia bibliografía, pero muchas especies faltan ser descriptas y varios problemas taxonómicos deben ser solucionados.

3. Pobremente conocido: áreas donde la literatura está esparcida e incompleta. Aquí la mayor parte de la fauna falta ser descripta y un gran número de especies será nueva para la ciencia. Los patrones de distribución no están claramente entendidos.

4. Mínimamente conocido: áreas donde la bibliografía consiste en descripciones taxonómicas esparcidas y registros aislados de distribución, muchos de ellos inadecuadamente sustentados.

Según esta clasificación, el conocimiento taxonómico sobre los octocorales en el Atlántico Sudoccidental está pobremente conocido para la costa sudamericana desde Trinidad y Tobago hasta el Río de la Plata, y mínimamente conocido para la costa sur del continente sudamericano (BAYER, 1981a).

Asimismo Bayer (1981b) hizo un listado de 1441 referencias bibliográficas mundiales sobre octocorales en el período comprendido entre los años 1469 y 1977, de los cuales sólo 110 fueron para el Atlántico Occidental (costa de EEUU, golfo de México, mar Caribe y costa este sudamericana); de estos trabajos la mayoría fueron para el Atlántico Norte, y sólamente 11 para las costas brasileras.

Los primeros registros taxonómicos para el litoral nordestino constan en los trabajos pioneros de Kukenthal (1903), Deichmamm (1936) y Tixier-Durivault (1970). A partir de la década del 80, fueron retomados los trabajos con estos antozoos (CASTRO, 1986, 1989; SILVEIRA, 1986). Los mayores conocimientos de la octofauna se obtuvieron a partir de la década del 90 con los trabajos de Castro (1990), Hetzel y

Castro (1994); Kelmo (1996), Medeiros (1998), Medeiros y Castro (1999), Amaral et al. (2000), Pérez y Silva (2001), Pérez (2002), Silva y Pérez (2002a y b) que estudiaron las especies del litoral nordestino, con nuevos registros y especies inéditas.

Al trabajar en la identificación y distribución de los organismos que ocurren en el litoral nordestino, se observaba una gran confusión cuanto a las especies identificadas y datos de distribución geográfica, com muchos vacios en las informaciones suministradas. Por esto fue verificada la necesidad de conocer la diversidad de los octocorales presentes en la costa nordestina; que serviria para informar acerca de los organismos que ocurren en el NE, evitando así desencuentro de informaciones, y auxiliando en la identificación de las posibles especies colectadas.

\section{RESULTADOS}

Através del análisis bibliográfico, fueron encontradas 59 especies descriptas para Brasil, dentro de las cuales, 25 se encuentran en el Nordeste (Tab. 1). 
Apesar de no poseer grupos de investigación, trabajando específicamente com la octofauna, en la región hasta el año 2000, la fauna de octocorales es relativamente conocida comparada con la de otras regiones del país (Tab. 2).

De las 25 especies citadas para la región Nordeste, el estado de Bahia es el que presenta la mayor cantidad (17), y el estado de Sergipe no presenta ningún registro en su costa (Tab. 2).

\section{CONCLUSIÓN}

De las 59 especies descriptas para el litoral brasilero 25 ocurren en el NE (42,37\%). De las 25 especies, 11 no ocurren en el resto del país, siendo cinco de ellas endémicas, Parerythropodium sp. Kelmo, 1996, Muriceopsis sp. Medeiros \& Castro, 1999, Plexaurella regia Castro, 1989, Primnoela polita Deichman, 1936 y Ceratoisis flexibilis (Pourtáles, 1868). También son registradas tres especies endémicas del Brasil con ocurrencia en otros estados: Muricea flama, Marques \& Castro, 1995, Plexaurella grandiflora Verril, 1912 y Phyllogorgia dilatata (Esper, 1806).

Tabela 1. Especies de octocorales encontradas en los estados de la región Nordeste de Brasil. BA, Bahia; SE, Sergipe; AL, Alagoas; PE, Pernambuco; RN, Rio Grande do Norte; CE, Ceará; PI, Piauí; MA, Maranhão; SC, Santa Catarina; SP, São Paulo; RJ, Rio de Janeiro; ES, Espírito Santo; PA, Pará; AP, Amapá; FN, Archipiélago de Fernando de Noronha; AT, Atol das Rocas; OR, Orden; TE, Telestacea; AL, Alcyonacea; GO, Gorgonacea; PE, Pennatulacea.

\begin{tabular}{|c|c|c|c|c|c|c|c|c|c|}
\hline \multirow[t]{2}{*}{ OR } & \multirow[t]{2}{*}{ ESPECIE } & \multicolumn{7}{|c|}{ ESTADOS DE LA REGIÓN NORDESTE } & \multirow[t]{2}{*}{ OTROS ESTADOS } \\
\hline & & BA SE & AL & $\mathrm{PE}$ & PB & $\mathrm{RN}$ & CE PI & MA & \\
\hline $\mathrm{TE}$ & Carijoa riisei & $\mathrm{X}$ & & $\mathrm{X}$ & & $\mathrm{X}$ & & $\mathrm{X}$ & SC, SP, RJ, ES, PA \\
\hline $\mathrm{AL}$ & Parerythropodium sp. & $\mathrm{X}$ & & & & & & & \\
\hline $\mathrm{AL}$ & Neospongodes atlántica & $\mathrm{X}$ & & & & $\mathrm{X}$ & & & \\
\hline $\mathrm{GO}$ & Chrysogorgia elegans & & & $\mathrm{X}$ & & & & & \\
\hline GO & Heterogorgia uatumani & $\mathrm{X}$ & & & & & & & SC, SP, RJ, ES, AP \\
\hline GO & Scleracis guadalupensis & $\mathrm{X}$ & & & & & & $\mathrm{X}$ & AP, PA, RJ \\
\hline GO & Thesea bicolor & $\mathrm{X}$ & & & & & & & ES \\
\hline GO & Muricea flama & $\mathrm{X}$ & & & & & & & ES \\
\hline GO & Muriceopsis sulphurea & $\mathrm{X}$ & $\mathrm{X}$ & $\mathrm{X}$ & $\mathrm{X}$ & & & & RJ, ES \\
\hline GO & Muriceopsis sp. & & & & $\mathrm{X}$ & $\mathrm{X}$ & $\mathrm{X}$ & $\mathrm{X}$ & \\
\hline GO & Plexaurella dichotoma & & & FN & & AR & & $\mathrm{X}$ & \\
\hline GO & Plexaurella grandiflora & $\mathrm{X}$ & $\mathrm{X}$ & $\mathrm{X}$ & $\mathrm{X}$ & & & & RJ, ES \\
\hline GO & Plexaurella regia & $\mathrm{X}$ & & & & & & & \\
\hline $\mathrm{GO}$ & Leptogorgia setacea & $\mathrm{X}$ & & & & & & & SC, SP, RJ, ES, PA \\
\hline GO & Leptogorgia miniata & & & & & & & $\mathrm{X}$ & RJ, PA \\
\hline GO & Leptogorgia punicea & $\mathrm{X}$ & & & & & & & $\begin{array}{r}\text { SC, PR, SP, RJ } \\
\text { Continua... }\end{array}$ \\
\hline
\end{tabular}


Tabela 1. Especies de octocorales encontradas en los estados de la región Nordeste de Brasil. BA, Bahia; SE, Sergipe; AL, Alagoas; PE, Pernambuco; RN, Rio Grande do Norte; CE, Ceará; PI, Piauí; MA, Maranhão; SC, Santa Catarina; SP, São Paulo; RJ, Rio de Janeiro; ES, Espírito Santo; PA, Pará; AP, Amapá; FN, Archipiélago de Fernando de Noronha; AT, Atol das Rocas; OR, Orden; TE, Telestacea; AL, Alcyonacea; GO, Gorgonacea; PE, Pennatulacea.. Continuación

\begin{tabular}{|c|c|c|c|c|c|c|c|c|c|c|c|}
\hline OR & ESPECIE & \multicolumn{9}{|c|}{ ESTADOS DE LA REGIÓN NORDESTE } & OTROS ESTADOS \\
\hline & & BA & SE & $\mathrm{AL}$ & $\mathrm{PE}$ & PB & RN & CE & PI & MA & \\
\hline GO & Leptogorgia sp. & $\mathrm{X}$ & & & & & & & & & \\
\hline GO & Olindagorgia gracilis & $X$ & & & & & & & & & \\
\hline GO & $\begin{array}{l}\text { Olindagorgia } \\
\text { marcgravii }\end{array}$ & & & & $\mathrm{X}$ & $\mathrm{X}$ & & & & & RJ, ES \\
\hline GO & Pacifigorgia elegans & & & & & & & & & $\mathrm{X}$ & \\
\hline GO & Phylogorgia dilatata & & & & FN & & $\mathrm{AR}$ & $\mathrm{X}$ & & & $\mathrm{ES}, \mathrm{RJ}$ \\
\hline GO & Ellisela barbadensis & $\mathrm{X}$ & & $\mathrm{X}$ & & & & & & & RJ, PA, AP \\
\hline GO & Primnoela polita & & & & $\mathrm{X}$ & & & & & & \\
\hline GO & Ceratoisis flexibilis & $\mathrm{X}$ & & & & & & & & & \\
\hline $\mathrm{PE}$ & Renilla muelleri & $\mathrm{X}$ & & $\mathrm{X}$ & & & & $\mathrm{X}$ & $\mathrm{X}$ & & RS, SC, SP, RJ, ES \\
\hline & Total de especies & 17 & 0 & 4 & 8 & 4 & 5 & 3 & 1 & 6 & \\
\hline
\end{tabular}

Tebela 2 - Cantidad de especies de octocorales en las diferentes regiones brasileras. $\mathrm{N}$, Norte; NE; Nordeste; SE, Sudeste; S, Sur.

\begin{tabular}{cc}
\hline REGIÓN & NÚMERO DE ESPECIES \\
\hline N & 17 \\
NE & 25 \\
SE & 32 \\
S & 14 \\
\hline
\end{tabular}

De las 25 especies registradas para la región nordeste, 21 pertenecen al Orden Gorgonacea, una a Telestacea, dos a Alcyonacea y una a Pennatulacea (Tab. 1); lo que revela la gran diversidad de los gorgonáceos en aguas tropicales. La baja abundancia de especies registradas de pennatuláceos se deve al hecho de que este grupo de animales habitan fondos blandos, y la mayoria de las colectas se realizan en áreas recifales, por lo cual, en regiones de mar abierto con fondos blandos, las colectas se restringen a las realizadas por expediciones científicas o de campañas de pesquerías bentónicas, lo cual torna totalmente accidental los registros para los octocorales de sustratos blandos.

El porcentaje obtenido para las especies que ocurren en la región NE es un poco engañoso, y merece un esclarecimiento. De las 25 especies registradas, 17 ocurren en el estado de Bahia, principalmente en el Archipiélago de Abrolhos, local sumamente estudiado por investigadores del sur-sudeste brasilero. Estas investigaciones encubren el real grado de conocimiento de la octofauna del NE, que excluyendo al estado de Bahia, representa 25,42\% (15 especies), colocando a la región dentro de la clasificación de Bayer (1981a), como pobremente conocida. El bajo número de especies citadas para otros estados nordestinos se deve exclusivamente a la falta 
de estudios y colectas en la región, ya que las 15 especies registradas para dichos estados (exclusive el de Bahia), se distribuyen de manera muy irregular, teniendo especies (sete) citadas para un solo estado, lo cual refleja que dichos registros son ocasionales o no forman parte de un estudio contínuo en el tiempo.

En la costa del estado de Sergipe, no fue encontrado ningún octocoral, lo que puede deberse a la falta de investigaciones en el lugar o por la influencia de la desembocadura del Río São Francisco. Otros estados de la región poseen su fauna poco o insuficientemente conocida, ya que no presentaban ningún especialista en la área, insuficiencia esta que será suplida debido a la existencia actual de investigadores formados o en formación en la propia región.

Actualmente están siendo desarrollados varios estudios con octocorales por el G.P.A. (Grupo de Pesquisa em Antozoários) de la UFPE, que dentro de sus líneas de investigación se encuentran la Sistemática, Taxonomía y Ecologia de los Octocorales del Noreste. Se espera encontrar nuevos organismos o nuevos registros para el litoral nordestino, pues considerando la conocida riqueza de la fauna de cnidarios de las zonas tropicales y la semejanza de la fauna del nordeste con la caribeña para otros grupos de cnidarios, además de la elevada diversidad de las regiones Sur y Sudeste de Brasil, es bastante probable que el número de especies presentes en la costa nordestina sea mayor que los registros actuales.

\section{REFERENCIAS}

AMARAL F. D., HUDSON M. M., COURA M. F. Levantamento da Cnidofauna do Arquipélago de São Pedro e São Paulo e do Parcel Manuel Luiz (MA). In: CONGRESSO BRASILEIRO DE ZOOLOGIA, 23, Cuiabá. Resumos... Cuiabá: Sociedade Brasileira de Zoologia, 2000. p.47.

BAYER, F. M., Octocorals from Surinam and adjacent coasts South America. Uitg. Natuurw.Studkring Suriname. (Stud. Fauna Suriname), v. 6, p.1-43, 1959.

BAYER, F. M. Status of Knowledge of octocorals of world seas. Pp 3-11. In: ACADEMIA BRASILEIRA DE CIÊNCIAS. Seminários de Biologia Marinha. São Paulo, 9 a 15 de Fevereiro de 1990. Rio de Janeiro, Academia Brasileira de Ciências. 383pp. 1981a.

BAYER, F. M. Recent advances in research on octocorals. pp19-102. In: ACADEMIA BRASILEIRA DE CIÊNCIAS. Seminários de Biologia Marinha. São Paulo, 9 a 15 de Fevereiro de 1990. Rio de Janeiro, Academia Brasileira de Ciências. 383pp. 1981b

BRITO, T. A. S. Taxonomic and ecological studies on Antartic octocorals of the genus Thouarella (Octocorallia, Primnoidae). 1993. 272 f. Tese (Doutorado em Ciências), University of Southampton, England.

CASTRO, C. B. Revisão sistemática dos octocorallia (Coelenterata, Anthozoa) dos recifes de Abrolhos, BA, Brasil. 1986. 89 f. Tese (Mestrado em Zoologia), Museu Nacional, Universidade Federal do Rio de Janeiro, Rio de Janeiro.

CASTRO, C. B. A new species of Plexaurella Valencienes, 1855 (Coelenterata, Octocorallia), from the Abrolhos reefs, Brazil. Ver. Brasil. Biol., v. 49, n.2, p. 597-603, 1989. 
CASTRO, C. B. Revisão taxonômica dos octocorallia (Cnidaria, Anthozoa) do litoral sulamericano: da foz do Rio Amazonas à foz do Rio da Prata. 1990. 342 f. Tese (Doutorado em Ciências), USP, São Paulo.

DEICHMAMM, E., The alcyonaria of the Western part of the Atlantic ocean. Memoirs of the museum of Comparative Zoology, v. 53, p. 253-30, 1936.

HETZEL, B.; CASTRO, C. B. Corais do Sul da Bahia. Rio de Janeiro: Nova Fronteira, 1994. 189 p.

KELMO, F. Uma nova espécie de Parerythropodium Kükenthal, 1916 (Octocorallia, Alcynacea) no Brasil. In: CONGRESSO BRASILEIRO DE ZOOLOGIA, 21, Porto Alegre. Resumos..., Porto Alegre: Sociedade Brasileira de Zoologia, 1996. p. 11.

KUKENTHAL, W. Über eine neue Nephthyidengattung aus dem südatlantischen Ocean. Zool. Anz., v. 26, n. 694, p. 272-275. 1903.

MEDEIROS. M. S. Octocorallia (Cnidária: Anthozoa) da plataforma continental e ilhas oceânicas brasileira: Famílias Paramuriceidae e Plexauridae. 1998. 157 f. Tese (Mestrado em Zoologia), Museu Nacional, UFRJ, Rio de Janeiro.

MEDEIROS, M. S.; CASTRO, C. B. Paramuriceidae e Plexauridae (Cnidaria, Octcorallia) do Brasil: Batimetria e Distribuição geográfica. Bol. Mus. Nac. n. 398, p.1-20, 1999.

PÉREZ, C. D. Taxonomia, Distribución y Diversidad de los Pennatulacea, Gorgonacea y Alcyonacea del mar epicontinental argentino y zonas de influencia. 1999. $254 \mathrm{f}$. Tese (Doutorado en Ciências Biológicas), Universidad Nacional de Mar del Plata, Argentina.

PEREZ, C. D. Octocorais (Cnidaria, Octocorallia) do litoral pernambucano (Brasil). In:

Diagnóstico da Biodiversidade de Pernambuco. Recife: Secretaria de Estado de Ciência, Tecnologia e Meio Ambiente de Pernambuco (SECTMA), Governo de Pernambuco e Editora Massangana, Tabarelli, M. \& Silva, J.M.P. (Eds.), 2002. Vol. II, p. 365-368.

PÉREZ, C. D., SILVA, B.T. Novas ocorrências de octocorais (Cnidaria, Anthozoa) do litoral pernambucano. In: ENCONTRO NORDESTINO DE ZOOLOGIA, 12, 2001, São Luis. Resumos... São Luis: Sociedade Nordestina de Zoologia, 2001, p.234.

SILVA, B. T.; PEREZ, C. D. Diagnose do conhecimento da fauna de octocorais (cnidaria, anthozoa) do litoral nordestino. In: CONIC-UFPE, 10, 2002, Recife. Resumos... Recife: Universidade Federal de Pernambuco, 2002a.

SILVA, B. T., PEREZ, C. D. Octocorais (Cnidaria, Anthozoa) do litoral maranhense, Brasil. Primeiro registro de Leptogorgia punicea (Milne-Edwards \& Haime, 1857). In: CONIC-UFPE, 10, 2002, Recife. Resumos... Recife: Universidade Federal de Pernambuco, 2002b.

SILVEIRA, F. L. Aspectos da biologia de Lophogorgia punicea (Milne-Edwards \& Haime, 1857) do Canal de São Sebastião, Estado de São Paulo. 1986. 211 f. Tese (Doutorado em Ciências), USP, São Paulo.

Tropical Oceanography, Recife: v. 30, n. 1, p. 15-22, 2002 
TIXIER-DURIVAULT, A. Octocoralliaires. Campagne de la Calypso au large des côtes Atlantiques de l’Amerique du Sud (1961-1962).I. Ann. Ins. Oceán., v. 47, p. 145-169, 1970. 